\title{
Development of an object-orientated database for wildfire modelling
}

\author{
C. J. Legg ${ }^{1}$, G. B. Pezzatti ${ }^{2}$, E. Rigolot ${ }^{3}$, O. Vigy ${ }^{3}$, I. Lecomte ${ }^{3}$, \\ A. Mårell ${ }^{3}$ \& V. Krivtsov ${ }^{1}$ \\ ${ }^{I}$ CECS, The University of Edinburgh, Scotland, UK \\ ${ }^{2}$ WSL Swiss Research Federal Institute, \\ Ecosystem Boundaries Research Unit, Switzerland \\ ${ }^{3}$ Mediterranean Forest Ecology Research Unit (UR629), \\ Fire Physics and Ecology Team, \\ National Institute for Agronomic Research (INRA), France
}

\begin{abstract}
Wild fires are a serious problem affecting many terrestrial ecosystems and causing substantial economic damage. The occurrence and intensity of wildfire depend (in part) on the availability and characteristics of fuel, which in natural landscapes is predominantly represented by plant litter and live vegetation. Therefore, a comprehensive collation of the information relevant to plant/litter fuel complexes, fire occurrences, and their responses to changes in environmental conditions is needed to enhance our understanding of this phenomenon. This paper provides a brief overview of modelling approaches used for analyses of wildfire fuels, and argues the case for an object orientated database of fuel complexes, which is being developed within the 'Fire Paradox' project (http://www.fireparadox.org/project_summary.php).
\end{abstract}

Keywords: wild fire, fuel structure, fuel models, allometric models, Fire Paradox.

\section{Introduction}

Wild fires are a serious problem affecting many terrestrial ecosystems and causing substantial economic damage. The occurrence and intensity of wildfires depend (in part) on the availability and characteristics of fuel, which in natural 
landscapes is predominantly represented by plant litter and live vegetation. Therefore, a comprehensive collation of the information relevant to plant/litter fuel complexes, fire occurrences, and their responses to changes in environmental conditions is needed to enhance our understanding of this phenomenon. This is necessary to design effective preventive and mitigating measures. This paper gives a brief overview of modelling approaches used for analyses of wildfire fuels, and illustrates the benefits of the object orientated database approach for the collation of this type of information.

\section{Fuel modelling in the terrestrial environment}

Prediction systems to describe forest fire behaviour require forest fuels to be described in a particular way with fuel characteristics represented by certain average values. The set of these representative values is called a "fuel model". Hence, knowledge of the vegetation and its structural dynamics is related to fire risk and is therefore indispensable for effective fuel control management.

There are a number of modelling approaches and model types relevant in this context, including, e.g.:

- Allometric models describing relationships between the major dimensions of plants, mostly shrubs and trees [1,2]

- Phytosociological Succession Models [3, 4] providing schemes and pathways of change from one vegetation type to another along post-fire gradients, or post-disturbance gradients

- Gap Models, describing vegetation dynamics initiated by disturbances $[5,6]$

- Vital Attribute Models [7, 8] relating to functional classification of traits determining the survival, regeneration and dispersal dynamics [9]

- Plant Architecture Models [10-12] predicting plant growth and form (and sometimes plant growth anomalies) throughout the plant's life, and in reaction to different stresses such as fire, competition, and management practices

- Landscape dynamics and mosaics models [13, 14] addressing the issues of e.g. species interactions, landscape engineering, and landuse planning.

A more detailed consideration of the above approaches here is precluded by the space constraints, and will be given elsewhere.

\section{FireParadox object orientated database}

\subsection{Fuel Data and OO approach}

We consider the fundamental unit of the database to be the 'Fuel Particle' with properties of size, moisture content, heat of combustion, etc. Fuel particles can be combined at increasing spatial scales to create a fuel complex (fig 1). Several different types of fuel complex are being represented using different 
organisational systems and hierarchies, including, for instance: models of individual plant crown architecture, building trees, populations and ecosystems from individual twigs and branches (the hierarchical 'Biological' model); models that are based on community-level structures such as vegetation strata without recognising individual plants (the 'Functional' model); or models that are based on discrete three-dimensional grids such as the cube model (the 'Spatial' approach). It is envisaged that the database described will ultimately form an important part of a free-access internet-based knowledge information system (fig. 2).

Type of data organisation

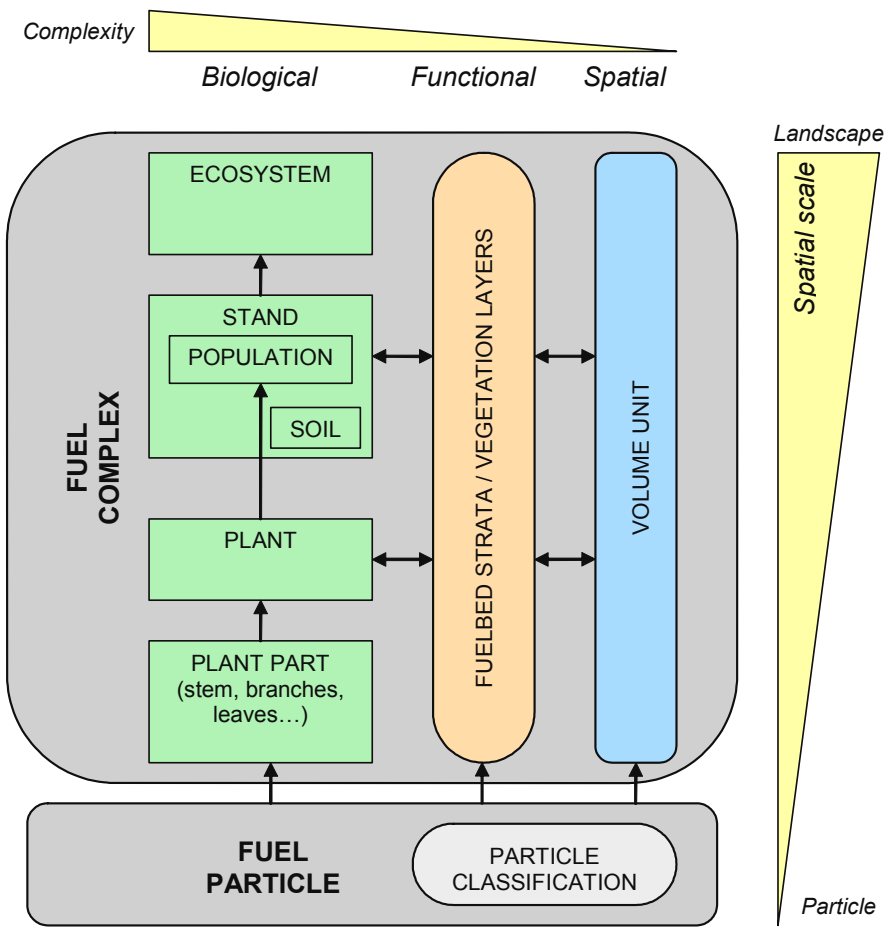

Figure 1: Conceptual structure of fuel data with examples of some of the different ways in which fuel compledes may be represented.

To date, the pilot database has been created with db4o (Java version) and successfully tested using both web-based (using the NextApp Echo2 library) and stand alone (using the SWING library) interfaces (fig. 3). An 'Allometric' module has been developed (using the scientific library of M.T. Flanagan (UCL)), allowing the user to carry out regression modelling (fig. 4) on selected fuel properties for different queries (e.g. volume fraction of small twigs against plant height and width). Work is currently underway to test the performance of these analyses using the INRA data, by comparing the outputs with the results 
42 Modelling, Monitoring and Management of Forest Fires I

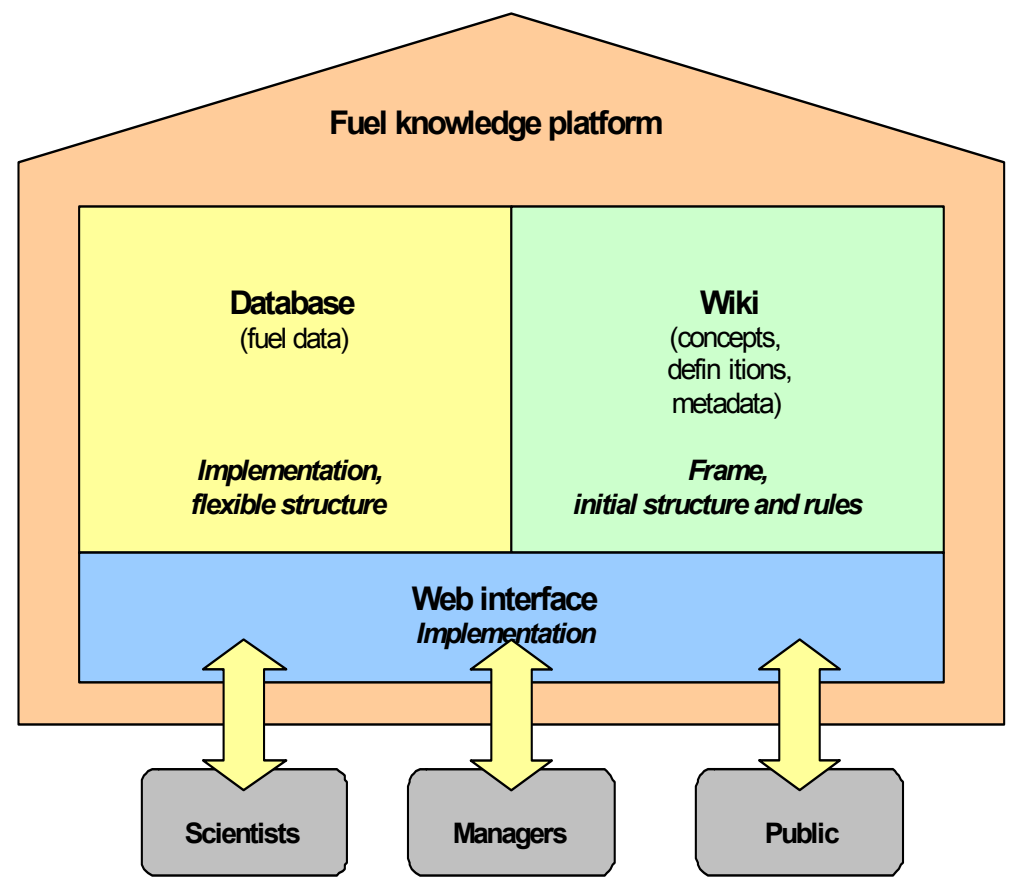

Figure 2: Overall structure of the application.

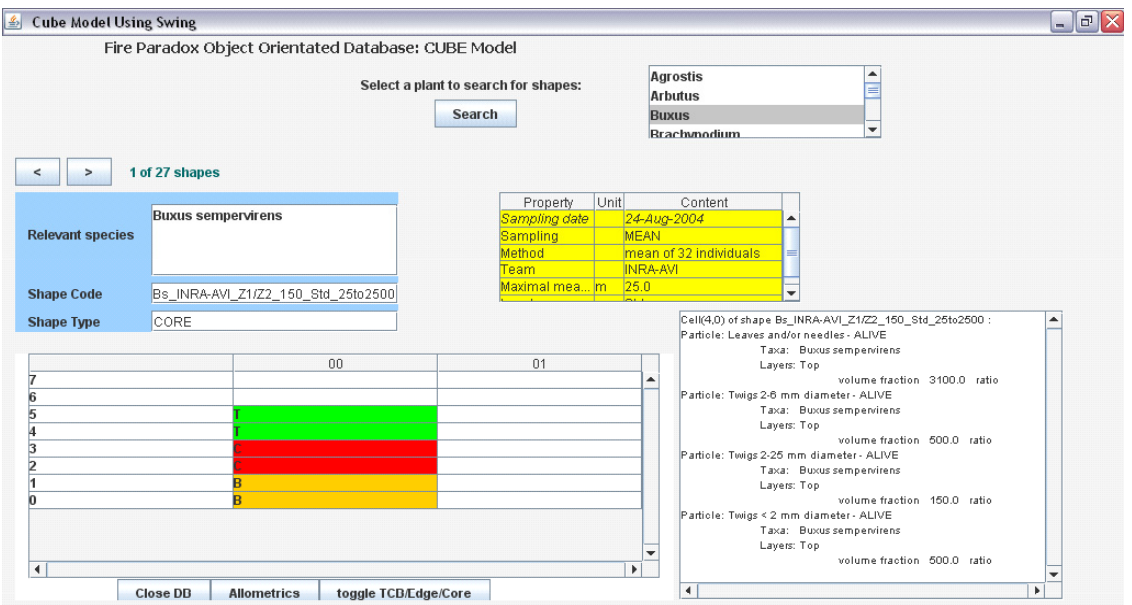

Figure 3: A view of the user interface for the stand alone application. 
previously obtained through the analysis of the primary data stored in a relational database (see Rigolot et al., this issue). Similar work is being undertaken on the Ulex data available in the University of Edinburgh (Higham and Legg, in preparation).

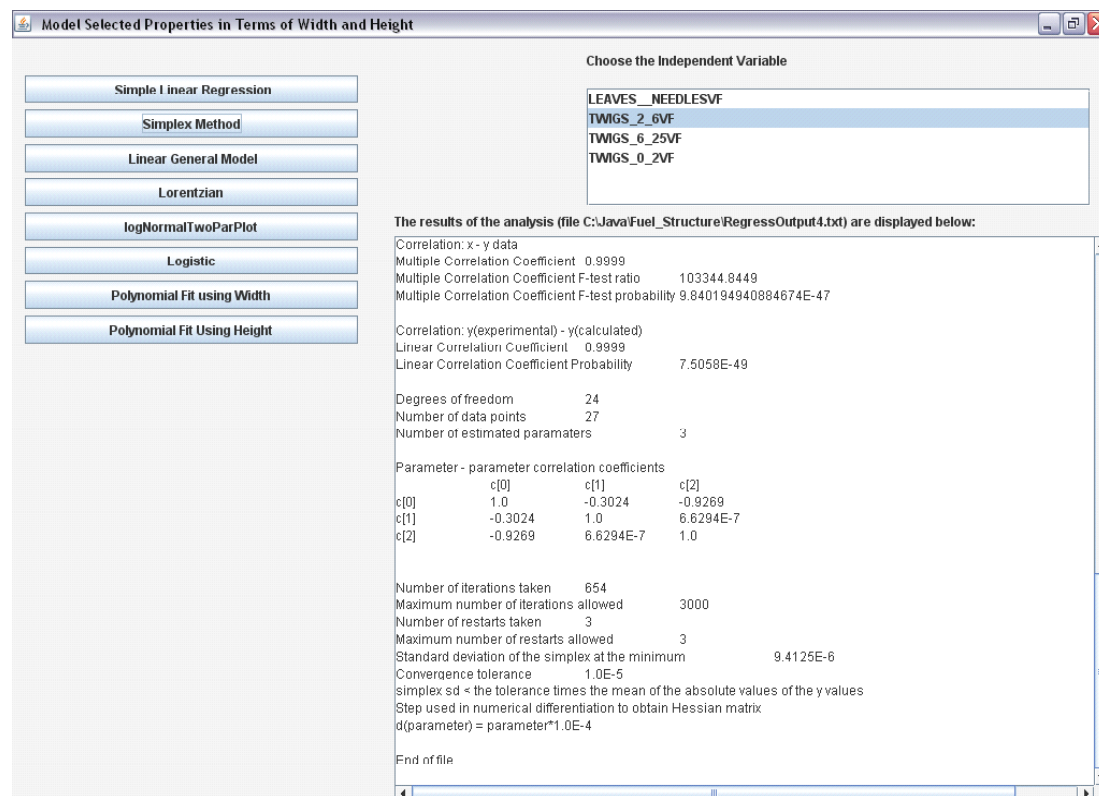

Figure 4: An example report of the 'Allometric' interface showing tools for regression analysis of fuel characteristics selected from the database.

We consider that the object-orientated approach provides a framework for defining data structures that is appropriate for our particular problem where we have a complex and hierarchical structure with a large number of variables. The relatively rigid relational database approach would result in a fragmented system with many missing values. It is, however, worth pointing out that the initial work related to the development of such a database, its underlying model, and the associated analysis tools and interfaces is relatively labour intensive (e.g. compared to relational databases), and requires from its developers both high levels of programming effort as well as expertise in relevant areas of biology and environmental science.

\subsection{Model structure}

For implementation of the fuel structures within the object-orientated database (OOD) we consider the fundamental unit of the database to be the 'Fuel Particle' with properties of size, moisture content, heat of combustion, etc. Fuel particles 
are combined in different ways at increasing spatial scales to create fuel complexes. This approach has the strong hierarchical complexity, but retains the compatibility between different representations through composition.

To facilitate the use of our database structure we have created a number of utility classes (e.g. Base) and a number of basic classes used by many other classes through inheritance (e.g. FProperties, Fuel, FKeySet) or implementation (e.g. AllometricFunction, FCollection). Arguably, the most important of those is FProperty. Each container (e.g. Cell, Plant, Shape, Particle) extends the base class Fuel, which, in turn, is a collection of FProperties. Each FProperty has a Content, a Definition, and may also have a collection of other FProperties (e.g. notes, metadata info, etc.). The Content is an object representing, e.g. an author, a bibliographic reference, or a collection of events.

It should be noted, that the database model structure is quite complex, and is characterised by a high level of interreferencing. Attribute collections could be assigned to objects by composition or inheritance (e.g. class Shape indirectly extends FKeySet, which implements FCollection, an interface extending Collection). As in Java only single inheritance is allowed, cases of multiple inheritance have had to be implemented with Java interfaces. As a rule we decided to use composition to add the fundamental attribute collection to fuel objects, and to use inheritance for the structural/hierarchical properties. This allows us a simpler check of the FProperty Domain, avoiding the use of parent references. Further information on the data model structure is available elsewhere [15].

\subsection{User interfaces and project structure}

To date, the pilot database has been created and successfully tested using both web-based (written in Echo2) and stand alone (written in SWING) interfaces (see an example in fig. 3).

For practical reasons, there are a number of interlinked modules, including e.g. FuelStructure (this is the core module containing the classes necessary for the implementation of the fuel/vegetation data model), FuelSwing (currently incorporating a simple interface and the Allometrics module), WebFuel (containing an interface to query the OOD via the web, in particular in relation to the INRA CUBE data). Each module project consists of a number of packages, and the links between the modules are implemented through imports of relevant packages and classes.

It should be noted, however, that as the study is still ongoing, the project structure is subject to changes, with the classes and whole packages being rearranged between different modules. For example, it is envisaged that the Allometric module may eventually be incorporated within the core (i.e. FuelStructure) module. On the other hand a number of auxiliary service classes (i.e. the ones used for formatting output, tokenising strings, liaising with 'on the fly compilers') may be separated within a separate module project.

It should be noted that the design of the database is such that the structure can be modified with minimum additional effort to avoid loss of current data. 


\section{Modelling allometric relationships}

An important part of the database design is the ability to analyse the stored data and to present the information at different levels of aggregation. The database engine includes the 'Allometric' module, incorporating a suite of mathematical techniques from the scientific library of M.T. Flanagan (UCL) (see fig. 3). The UI allows the user to carry out regression modelling on the selected fuel properties for any specific query (e.g. volume fraction of small twigs against height and width of plants).

AllometricFunction is an important Java interface within the Allometrics module, extending RegressionFunction of Flanagan. It has provisions to create composite equations using specific instances of a class of simple mathematical functions, e.g. power, logarithmic, trigonometric. An instance of AllometricFunction may be compiled at run time, using the capabilities of the Janino compiler.

Work is currently underway to test the performance of the allometric analyses by comparing outputs with the results previously obtained through analysis of the primary data stored in spreadsheets and/or a relational database.

\section{Fuel manager}

The Fire Paradox Fuel Manager (FPFM) has been developed as a module in Capsis, a free platform dedicated to host a wide range of forest dynamics and stand growth models [16]. It creates - in user friendly manner - the fuel input files of physically-based fire simulation models. These input files describe the composition and the structure of the fuel complex taking into account the physical properties of various components of the different layers (trees, shrubs, herbs and litter) composing the vegetation scene. Data related to fuel description are stored in a database with a structure that has been designed on the basis of an inventory of both available fuel data (using the INRA cube method) as well as expected 3D fuel data from other project partners.

FPFM is, on one hand, a management tool for manipulating fuel complexes and on the other hand, a software that enables fire simulations and the modelling of post fire vegetation succession. Currently, the work carried out in collaboration with the Capsis developers has related to the Graphical User Interfaces (GUI), e.g. development of $3 \mathrm{D}$ visualisation viewers and tools to interact dynamically with the vegetation objects. A number of manipulative functionalities have been implemented in conjunction with the FireParadox object-orientated database, e.g. the generation of a vegetation scene with welldescribed fuel individuals. The next important step will be the generation of fuel input files for the fire behaviour model.

\section{Concluding remarks}

The FireParadox Object Orientated database approach presented here has been implemented as a proof of concept. It appears to be very promising, and is deemed to be potentially useful in a wide range of practical applications. 
It should be noted, however, that the development of the system presented here requires a combination of extensive programming effort, good statistical and mathematical modelling skills, and high level of expertise in the relevant areas of environmental sciences, which is not always adequately appreciated by the funding bodies. Consequently, the development of the pilot system presented here as a proof of concept, has been labour intensive, and the actual requirements for resources has exceeded our initial expectations. Future developments and applications of this approach will, therefore, be subject to further funding.

\section{Acknowledgement}

We acknowledge the European Commission's Sixth Framework Programme for funding the research, which is part of the Fire Paradox project.

\section{References}

[1] Gong, W.K. and J.E. Ong, The Use of Demographic-Studies in Mangrove Silviculture. Hydrobiologia, 1995. 295(1-3): p. 255-261.

[2] Yang, Y.S., et al., Soil respiration and carbon balance in a subtropical native forest and two managed plantations. Plant Ecology, 2007. 193(1): p. 71-84.

[3] Lee, W.G., R.B. Allen, and P.N. Johnson, Succession and Dynamics of Gorse (Ulex-Europaeus L) Communities in the Dunedin Ecological District South-Island, New-Zealand. New Zealand Journal of Botany, 1986. 24(2): p. 279-292.

[4] Zavala, M.A. and E. Zea, Mechanisms maintaining biodiversity in Mediterranean pine-oak forests: insights from a spatial simulation model. Plant Ecology, 2004. 171(1-2): p. 197-207.

[5] Raventos, J., J. Segarra, and M.F. Acevedo, Growth dynamics of tropical savanna grass species using projection matrices. Ecological Modelling, 2004. 174(1-2): p. 85-101.

[6] Scheller, R.M. and D.J. Mladenoff, An ecological classification of forest landscape simulation models: tools and strategies for understanding broadscale forested ecosystems. Landscape Ecology, 2007. 22(4): p. 491-505.

[7] Bradstock, R.A. and B.J. Kenny, An application of plant functional types to fire management in a conservation reserve in southeastern Australia. Journal of Vegetation Science, 2003. 14(3): p. 345-354.

[8] Cornelissen, J.H.C., et al., A handbook of protocols for standardised and easy measurement of plant functional traits worldwide. Australian Journal of Botany, 2003. 51(4): p. 335-380.

[9] Noble, I.R. and H. Gitay, A functional classification for predicting the dynamics of landscapes. Journal of Vegetation Science, 1996. 7(3): p. 329336.

[10] Acevedo, M.F. and J. Raventos, Growth dynamics of three tropical savanna grass species: an individual-module model. Ecological Modelling, 2002. 154(1-2): p. 45-60. 
[11] Keane, R.E., K.C. Ryan, and S.W. Running, Simulating effects of fire on northern Rocky Mountain landscapes with the ecological process model FIRE-BGC. Tree Physiology, 1996. 16(3): p. 319-331.

[12] Kurth, W., Morphological Models of Plant-Growth - Possibilities and Ecological Relevance. Ecological Modelling, 1994. 75: p. 299-308.

[13] Busing, R.T., et al., Forest dynamics in Oregon landscapes: Evaluation and application of an individual-based model. Ecological Applications, 2007. 17(7): p. 1967-1981.

[14] Pausas, J.G., Simulating Mediterranean landscape pattern and vegetation dynamics under different fire regimes. Plant Ecology, 2006. 187(2): p. 249259.

[15] Pezzatti, G.B., et al. An object orientated fuel database within the knowledge information system of the Fire Paradox project. . in 7th European Ecological Modelling Conference. 2007. Trieste.

[16] de Coligny, F., et al. CAPSIS: Computer-Aided Projection for Strategies In Silviculture: Open architecture for a shared forest-modelling platform. in Connection between Forest Resources and Wood Quality: Modelling Approaches and Simulation Software. Fourth workshop, IUFRO Working Party S5.01.04, 8-15/09/2002. 2004. Harrison Hot Springs Resort, British Columbia, Canada: Nancy, France: LERFoB INRA-ENGREF. 